\title{
Behavioral and neurobiological changes in C57BL/6 mouse exposed to cuprizone: effects of antipsychotics
}

\author{
Haiyun Xu ${ }^{1}{ }^{*}$, Hong-Ju Yang ${ }^{1}$, Bryan McConomy ${ }^{2}$, Ronald Browning ${ }^{2}$ and Xin-Min Li ${ }^{3}$ \\ 1 Department of Anatomy, Southern Illinois University Carbondale, Carbondale, IL, USA \\ 2 Department of Physiology, Southern Illinois University Carbondale, Carbondale, IL, USA \\ ${ }^{3}$ Department of Psychiatry, University of Manitoba, Winnipeg, MB, Canada
}

\section{Edited by:}

Julietta U. Frey, Leibniz Institute for

Neurobiology, Germany

Reviewed by:

Satoru Otani, University of Paris VI,

France

Jorge A. Bergado, Department of

Experimental Neurophysiology, Cuba

\section{${ }^{*}$ Correspondence:}

Haiyun Xu, Department of Anatomy, Southern Illinois University Carbondale, 1135 Lincoln Dr., Carbondale, IL 62901, USA.

e-mail: hxu@siumed.edu
Recent human studies suggest a role for altered oligodendrocytes in the pathophysiology of schizophrenia. Our recent animal study has reported some schizophrenia-like behaviors in mice exposed to cuprizone (Xu et al., 2009), a copper chelator that has been shown to selectively damage the white matter. This study was to explore mechanisms underlying the behavioral changes in cuprizone-exposed mice and to examine effects of the antipsychotics haloperidol, clozapine and quetiapine on the changes in the mice. Mice given cuprizone for 14 days showed a deficit in the prepulse inhibition of acoustic startle response and higher dopamine in the prefrontal cortex (PFC), which changes were not seen in mice given cuprizone plus antipsychotics. Mice given cuprizone for 21 days showed lower spontaneous alternations in Y-maze, which was not seen in mice treated with cuprizone plus the antipsychotics. Mice given cuprizone for 28 days displayed less social interactions, which was not seen in mice given cuprizone plus clozapine/ quetiapine, but was seen in mice given cuprizone plus haloperidol. Mice given cuprizone for 42 days showed myelin sheath loss and lower myelin basic protein in PFC, caudate putamen, and hippocampus. The white matter damage in PFC was attenuated in mice given cuprizone plus clozapine/haloperidol. But the white matter damage in caudate putamen and hippocampus was only attenuated by clozapine and quetiapine, not by haloperidol. These results help us to understand the behavioral changes and provide experimental evidence for the protective effects of antipsychotics on white matter damage in cuprizone-exposed mice.

Keywords: antipsychotics, schizophrenia model, behavior, oligodendrocytes, MBP, mouse

\section{INTRODUCTION}

Schizophrenia is one of the most severe psychiatric disorders, affecting about $1 \%$ of the worldwide population. The symptoms of the disorder are usually classified as positive, negative, and cognitive symptoms (Carpenter and Buchanan, 1994). Positive symptoms include hallucinations, delusions, and thought disorganization. Negative symptoms are a group of deficits comprising many dimensions, such as diminished emotional response, social withdrawal, blunted affect, poverty of speech, and lack of energy. Cognitive symptoms include deficits in attention, executive function, and memory. Patients usually come to clinical attention because of their positive symptoms.

Although the etiology and fundamental pathophysiology of schizophrenia remain unclear, significant advances have been made in recent human studies. First, neuroimaging studies have shown white matter abnormalities and enlarged ventricles in schizophrenic brains (Ardekani et al., 2003; Davis et al., 2003; Kubicki et al., 2005). Second, genome-wide expression analyses have shown dysregulation of genes related to oligodendrocyte (OL) function and myelination in schizophrenia (Hakak et al., 2001; McCullumsmith et al., 2007). Third, patients with white matter disorders experience a host of psychotic symptoms (Kohler et al., 1988; Hyde et al., 1992; Bassett et al., 2003). These previous studies suggest a putative role for altered OLs in the pathophysiology of schizophrenia. However, experimental evidence for this novel hypothesis has been scarce.
Among efforts to establish an appropriate animal model to test this novel hypothesis, two most recent studies reported promising results, using an established animal model featured with selective damage in the white matter of the central nervous system (CNS) caused by cuprizone (CPZ) (Matsushima and Morell, 2001; Yang et al., 2009), a copper chelator. In the first study by our lab, C57BL/6 mice given CPZ showed schizophrenia-like behaviors including deficits in prepulse inhibition (PPI) of acoustic startle reflex, less social interaction, and impaired spatial working memory. These animals also showed evident myelin sheath loss and decrease in the number of OLs in the brain (Xu et al., 2009). In the second study adolescent rats exposed to $\mathrm{CPZ}$ showed decreased expression of mRNA transcripts encoding oligodendroglial proteins within the medial prefrontal cortex (PFC); these rats also displayed a specific deficit in the ability to shift between perceptual dimensions in the attention set-shifting task (Gregg et al., 2009), a PFC-mediated behavioral paradigm modeled after the Wisconsin Card Sorting Test (Franke et al., 1992). These studies suggest that the CPZ-exposed mice/rats are potential animal models of schizophrenia to test the OL hypothesis.

For the first time, this study examined effects of some antipsychotics on the behavioral abnormalities and neurobiological changes seen in C57BL/6 mice exposed to cuprizone. Our results provided experimental evidence supporting a role for altered OLs in the pathophysiology and treatment of schizophrenia. The drugs 
tested are haloperidol (HAL), clozapine (CLZ), and quetiapine (QUE). HAL is a prototype of the first-generation antipsychotics, while CLZ and QUE are second-generation antipsychotics (SGAs). These drugs are being used for patients with schizophrenia, but have different therapeutic actions and mechanisms. HAL is effective in reducing psychotic symptoms, but has poor effects on the negative and cognitive symptoms (Ellenbroek, 1993), while CLZ and QUE are effective in improving negative symptoms and cognitive deficits (Kane et al., 1988; Harvey and Keefe, 2001; Woodward et al., 2005). HAL has a high affinity for D2 receptors (Seeman, 2006) whereas CLZ and QUE are broad-acting agents, which not only exhibit affinity for a variety of DA receptors, but also for other metabotropic receptors, e.g. those for serotonin (5-HT2A and 5-HT1), norepinephrine (NE), acetylcholine and histamine (Meltzer, 1995; Seeman, 2002; Reynolds, 2004). Blocking D2 receptors in mesolimbic areas is believed to be responsible for the therapeutic effects on the positive symptoms (Seeman, 2006). However, the mechanisms underlying the improvement of negative and cognitive symptoms by SGAs remain to be elucidated.

\section{MATERIALS AND METHODS CHEMICALS AND REAGENTS}

CPZ, HAL, and CLZ were purchased from Sigma-Aldrich (St. Louis, MO,USA). QUE was provided by AstraZeneca Canada (Mississauga, Ontario). CPZ was mixed into a standard powdered rodent chow at the final concentration $0.2 \%(\mathrm{w} / \mathrm{w})$. All the antipsychotics were freshly prepared (dissolved in $0.8 \%$ glacial acetic acid in saline and $\mathrm{pH}$ value was adjusted to 6.8 ) and administered intraperitoneally (i.p; $1 \mathrm{ml} / 100$ g body weight) once a day. On the behavioral test day, antipsychotics were administered after finishing behavioral tests. Based on previous studies (Xu et al., 2002; Bai et al., 2003, 2004) $1 \mathrm{mg} / \mathrm{kg} /$ day of HAL, $10 \mathrm{mg} / \mathrm{kg} /$ day of CLZ and QUE were used in this study. The primary antibody to MBP and secondary antibody were purchased from Millipore (Temecula, CA, USA), antibody to $\beta$-actin from Sigma-Aldrich, the ABC kits from Vector Labs (Burlingame, CA, USA), and the ECL plus ${ }^{\mathrm{TM}}$ kit from Amersham Biosciences (Buckinghamshire, UK).

\section{EXPERIMENTAL DESIGN}

Male C57BL/6 mice (6-weeks old, 20 to $22 \mathrm{~g}$ ) were purchased from Charles River Laboratories (Wilmington, MA, USA). After an acclimatization period of 10 days, the C57BL/6 mice were given $0.2 \%$ by weight CPZ in the standard powdered rodent chow for 14-42 days, during which age-matched mice received the standard chow without CPZ and were used as controls. Three independent experiments were performed for HAL, CLZ, and QUE, respectively. Each experiment consisted of four animal groups designed to examine effects of CPZ on animals' behaviors and white matter and effects of the antipsychotics in mice without or with CPZ-exposure. For example, HAL experiment included CNT, $\mathrm{CPZ}, \mathrm{HAL}$, and $\mathrm{CPZ}+\mathrm{HAL}$ groups. CNT group mice ate normal rodent chow without $\mathrm{CPZ}$ and received no $\mathrm{HAL}$; $\mathrm{CPZ}$ group mice ate $\mathrm{CPZ}$-containing rodent chow and received no HAL; HAL group mice ate normal rodent chow without $\mathrm{CPZ}$ but received HAL treatment $(1 \mathrm{mg} / \mathrm{kg} /$ day, i.p.); $\mathrm{CPZ}+\mathrm{HAL}$ group mice ate $\mathrm{CPZ}$ containing rodent chow and received HAL treatment. The experimental period was 42 days, during which mice were subjected to behavioral tests at indicated time points. For high-performance liquid chromatography (HPLC) analysis, two additional experiments were performed, in which the same treatments $(\mathrm{CPZ}$ ingestion and antipsychotics administration) continued for 12 and 21 days, respectively. Each of the experiments consisted of eight animal groups (CNT, CPZ, HAL, CLZ, QUE, CPZ+HAL, $\mathrm{CPZ}+\mathrm{CLZ}$, and CPZ+QUE; 5-8 mice/group). All animal procedures in this study were in accordance with the National Institute of Health Guide for the Care and Use of Laboratory Animals and were approved by the Animal Care and Use Committee of Southern Illinois University Carbondale.

\section{PREPULSE INHIBITION TEST}

PPI refers to the inhibition of a startle reflex produced by preceding the startling stimulus, or pulse, with a weak prepulse stimulus. This test provides an operational measure of sensory gating of subjects. In our recent study, CPZ-exposed mice showed PPI deficits on 14th and 21st days after CPZ-exposure (Xu et al., 2009). In this study PPI test was performed on 14th day after CPZ-exposure. As described in our recent study (Xu et al., 2009), each mouse was placed into a small Plexiglas cylinder within a large sound-attenuating chamber (San Diego Instruments, CA, USA). The cylinder was seated upon a piezoelectric transducer, which allows vibrations to be quantified and displayed on a computer. The background sound levels (74-75 dB) and calibration of the acoustic stimuli were confirmed with a digital sound level meter. After a 5-min habituation period, PPI test sessions were conducted. Each session started and ended with five startle trials ( $40 \mathrm{~ms} ; 120 \mathrm{~dB}$ ), respectively. Between the starting and ending startle trials, there were eight identical blocks consisting of the following five trials: a no-stimulus trial, a startle trial, and three prepulse-startle trials, each of which had a pre-pulse stimulus (3, 6, or $12 \mathrm{~dB}$ above the background sound levels) prior to a startle stimulus (100 ms after the prepulse). The average inter-trial interval was $15 \mathrm{~s}$. Measures were taken of the startle amplitude for each trial, defined as the peak response during a 65-ms sampling window starting from the onset of a startle stimulus. Levels of PPI at each prepulse sound level were calculated as $100 \times(1$-averaged response amplitude in trials with a prepulse stimulus and startle stimulus/averaged response amplitude in trials with the startle stimulus alone).

\section{Y-MAZE TEST}

The Y-maze is a simple two-trial recognition test for measuring spatial recognition memory. In a previous study (Oades et al., 1985), amphetamine reduced alternation dose-dependently and HAL pretreatment inhibited this effect, suggesting that this paradigm is useful for studying spatial working memory in animal models of schizophrenia. In our recent study, CPZ-exposed mice showed lower spontaneous alternation in Y-maze on 14th, 21st, 28th, 35th, and 42nd days after CPZ-exposure (Xu et al., 2009). In this study Ymaze test was performed on 21 st and 42 nd days after CPZ-exposure. As described in our recent study (Xu et al., 2009), each mouse was placed at the end of one arm of a symmetrical Y-maze and allowed to move freely through the maze during an 8 -min test period. The total number and series of arm entries were recorded. The number of overlapping entrance sequences (e.g., ABC, BCA) defines the number of spontaneous alternations. 


\section{SOCIAL INTERACTION TEST}

In our recent study, CPZ-exposed mice showed less social interaction on 28th, 35th, and 42nd days after CPZ-exposure (Xu et al., 2009). In this study social interaction test was performed on 28th day after CPZ-exposure. As described in our recent study (Xu et al., 2009), pairs of unfamiliar mice (that had been housed separately) of the same experimental group were placed in the center of an openfield box $(56 \mathrm{~cm} \times 56 \mathrm{~cm} \times 31 \mathrm{~cm})$ about $10 \mathrm{~cm}$ apart. A video camera was placed above the open-field box to monitor the animals' movement. During a 10 min test period, the social interaction of the animals was defined when the tested mice were closer than $5 \mathrm{~cm}$ (between the center point of the back of a mouse and another one) for at least $0.2 \mathrm{~s}$. Each mouse was used only one time. The results were recorded and analyzed automatically by the video tracking program SMART, which counted the amount of time spent near another mouse for two mice simultaneously. Although this method only measures closeness of subjects examined, it has been used in previous studies and the closeness is believed to reflect social interactions (Shi et al., 2003; Egashira et al., 2007), which is an animal correlate of social withdrawal seen in patients with schizophrenia (Weinberger, 1987; Abi-Dargham et al., 2000). Social behavioral deficit has been reported in various animal models of schizophrenia (Shi et al., 2003; Boucher et al., 2007; Lazar et al., 2008).

\section{IMMUNOHISTOCHEMISTRY AND IMAGE ANALYSIS}

For immunohistochemical staining, animals were deeply anesthetized with chloral hydrate $(400 \mathrm{mg} / \mathrm{kg} \mathrm{ip}$ ) and perfused through the ascending aorta with $0.1 \mathrm{M}$ phosphate buffered saline (PBS; $\mathrm{pH}$ 7.4), followed by $4 \%$ paraformaldehyde in PBS. Their brains were then removed and immersed in the same fixative overnight, followed by cryo-protection in $25 \%$ sucrose at $4^{\circ} \mathrm{C}$ for $24-48 \mathrm{~h}$. Serial coronal sections $(25 \mu \mathrm{m})$ of the brains were cut using a sliding microtome (Lipshaw, Detroit, MI, USA) and collected in six well plates containing 0.01-M PBS. One set of the sections was used to assess the MBP-like immunoreactivity using the protocol as described previously (Yang et al., 2009). For quantitative analysis, five sections from each brain region ( $\mathrm{CP}$ and hippocampus) of each animal were digitally recorded using a DMI-4000B microscope (Leica, Germany) equipped with a digital capture system. The optical densities of MBP-like immunoreactivity and positive stained area in the target regions were measured using the ImagePro Plus software (Version 6.0, Media Cybernetics, Inc., Bethesda, MD, USA) under the same conditions. In a same experiment, the value of the positive stained area in the target regions of CNT group was used as the standard for the normalization of the values of other groups.

\section{WESTERN-BLOT ANALYSIS}

Under deep anesthesia mice were sacrificed, their brains were rapidly removed, and specific brain regions (PFC, CP, and hippocampus) were dissected out and stored at $-80^{\circ} \mathrm{C}$ until use. Western-blot was performed to assess the amount of MBP in each brain region using the antibody to MBP as described previously (Zhang et al., 2008). Immunoreactive bands were visualized using a UVP Biodocit $^{\mathrm{TM}}$ system (UVP, Inc., Upload, CA, USA) and measured with the Image J software (NIH, Bethesda, MD, USA). The product of optical density and area of each band was calculated. For each lane, there is a ratio of MBP product/ $\beta$-actin product. In a same experiment, the ratio of CNT group was used as the standard for the normalization of the ratios of other groups.

\section{HIGH-PERFORMANCE LIOUID CHROMATOGRAPHY}

In our recent study, CPZ-exposed mice showed changes in DA and $\mathrm{NE}$ on 14 th and 21 st days after CPZ-exposure, respectively (Xu et al., 2009). Therefore, the same time points were chosen for the measurement of these two neurotransmitters. Mice were decapitated and the PFC was dissected out of the brain and frozen at $-80^{\circ} \mathrm{C}$ until use. DA and NA levels in PFC of mice were measured using HPLC method as described in our recent study (Xu et al., 2009).

\section{DATA ANALYSIS}

Quantitative data were analyzed by two-way analysis of variance considering CPZ treatment and antipsychotic administration as two main factors, followed by Tukey's multiple comparisons. When a $p$-value was less than 0.05 , the difference/effect was considered significant.

\section{RESULTS \\ ANTIPSYCHOTICS BLOCK THE DEFICIT OF CPZ-EXPOSED MICE IN THE PREPULSE INHIBITION}

In the HAL experiment (Figure 1A), when the prepulse of $3 \mathrm{db}$ was applied, no significant effect was found by two-way ANOVA $\left[F_{(3,39)}=0.77 ; p=0.5\right]$. And no differences were found between any two groups. When the prepulse of $6 \mathrm{db}$ was applied, two-way $\operatorname{ANOVA}\left(F_{(3,39)}=4.27 ; p=0.024\right)$ revealed a significant interaction between the two main factors ( $\mathrm{CPZ}$ and HAL treatments). And significant differences were also found in the comparisons of CNT and CPZ, CNT and HAL, as well as CPZ and CPZ+HAL (individual $F$ and $p$ values were not presented). When the prepulse of $12 \mathrm{db}$ was applied, two-way ANOVA $\left(F_{(3,39)}=4.07 ; p=0.017\right)$ revealed a significant interaction between the two main factors. And significant differences were also found in the comparisons of $\mathrm{CNT}$ and $\mathrm{CPZ}$, as well as $\mathrm{CPZ}$ and $\mathrm{CPZ}+\mathrm{HAL}$ (individual $F$ and $p$ values were not presented). Similar results were also found in CLZ (Figure 1B) and QUE (Figure 1C) experiments. But unlike HAL, CLZ and QUE did not caused a PPI deficit.

\section{DIFFERENT EFFECTS OF ANTIPSYCHOTICS ON THE ABNORMAL PERFORMANCE OF CPZ-EXPOSED MICE IN Y-MAZE}

In the HAL experiment (Figures 2A,B), CPZ-exposure for 21 days lowered $(p=0.001)$ the spontaneous alternation in Y-maze test. But no differences were found between other groups. HAL alone decreased the number of arm entries compared to CNT, suggesting a sedative effect of this drug on the mice. When CPZ-exposure was extended for 42 days, a more significant $(p=0.0002)$ lower spontaneous alternation was found. Moreover, lower spontaneous alternations were also found in HAL and CPZ + HAL groups, suggesting that HAL alone had an adverse effect on this behavioral performance and that this drug was unable to prevent mice from the effect of $\mathrm{CPZ}$ on this performance. And the sedative effect was found in HAL and CPZ + HAL groups. In the CLZ experiment (Figures 2C,D), CPZ-exposure for 21 days lowered the spontaneous alternation $(p=0.011)$. And lower spontaneous alternation was also found in the CPZ + CLZ group but was not significant 


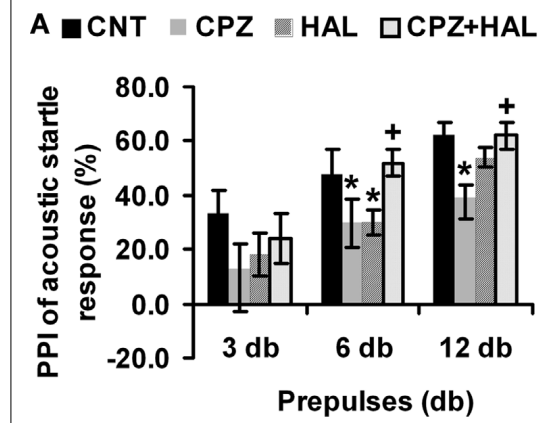

FIGURE 1 | Effects of antipsychotics on the CPZ-induced deficits in PPI test. Control and experimentally treated C57BL/6 mice were subjected to PPI test on the same day (14th day after CPZ-exposure). (A)The data of the HAL experiment. (B) The data of the CLZ experiment. (C) The data of the QUE experiment. Data were expressed as $\mathrm{M} \pm \mathrm{SEM}$ ( $n=6$ to 12/group). CNT, Control group; CPZ,
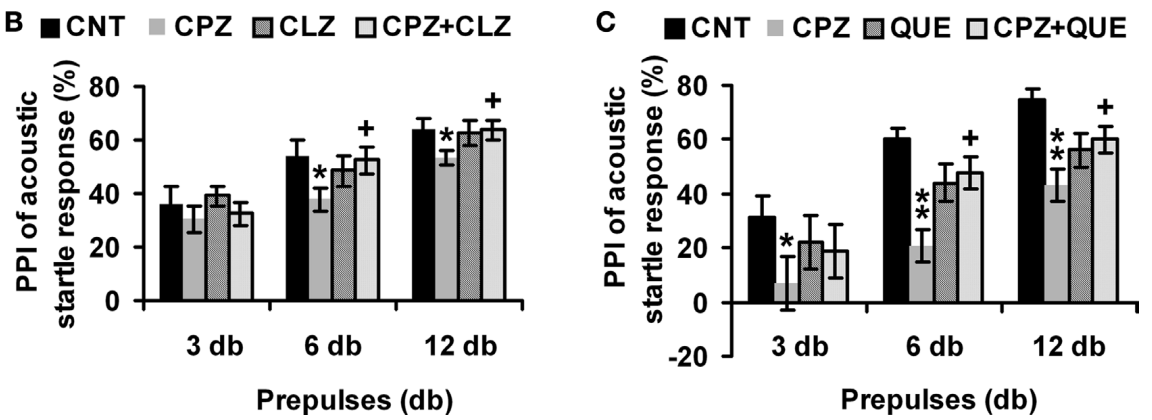

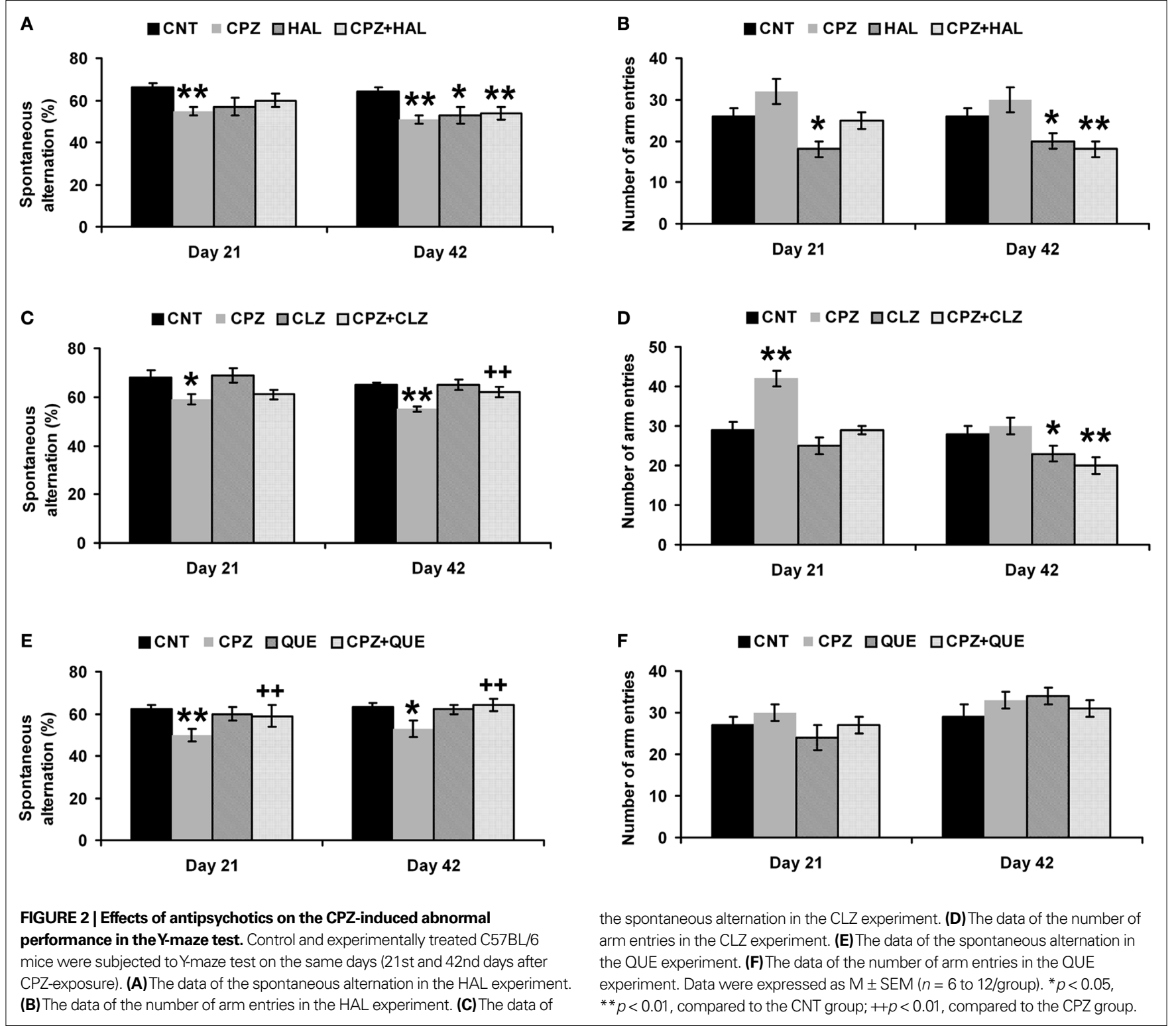


$(p=0.064)$. Interestingly, mice exposed to CPZ for 21 days showed more number of arm entry in this experiment. But, this increase was not found in the CPZ + CLZ group. When CPZ-exposure was extended for 42 days, the CPZ-induced lower spontaneous alternation was not found in the CPZ + CLZ group. Moreover, mice exposed to CLZ for 42 days had fewer number of arm entries $(p=0.02)$. In the QUE experiment (Figures 2E,F), CPZ-induced lower spontaneous alternation was not found in the CPZ + QUE group (Figure 2E); but, the treatments did not affect the number of arm entries in QUE and CPZ + QUE groups (Figure 2F).

\section{CLZ AND QUE, BUT NOT HAL, BLOCK THE ABNORMAL PERFORMANCE OF CPZ-EXPOSED MICE IN SOCIAL INTERACTION}

In the first 20-30 s, the tested mice moved about but avoided the partner in the open field. Then, avoiding behaviors disappeared and social interactions became frequent, including sniffing the partner's body, grooming the partner, and mounting the partner. In the HAL experiment (Figure 3A), two-way ANOVA revealed a significant interaction between CZP and HAL treatments $\left[F_{(3,23)}=16.59\right.$, $p<0.001]$. Post hoc comparisons found significant differences between CNT and other groups (CPZ, HAL, and CPZ+HAL), but not between any other two groups. In the CLZ experiment (Figure 3B), two-way ANOVA revealed a significant interaction between CZP and CLZ treatments $\left[F_{(3,23)}=5.98, p<0.01\right]$. Post hoc comparisons found a significant difference between CNT and $\mathrm{CPZ}$ groups. The difference between $\mathrm{CPZ}$ and $\mathrm{CPZ}+\mathrm{CLZ}$ groups was also significant. But, no differences were found between any other two groups. In the QUE experiment (Figure 3C), two-way ANOVA revealed a significant interaction between CZP and QUE treatments $\left[F_{(3,23)}=3.85, p<0.05\right]$. Post hoc comparisons found a significant difference between $\mathrm{CNT}$ and $\mathrm{CPZ}$ groups. But, no differences were found between any other two groups.

\section{ANTIPSYCHOTICS BLOCK THE CHANGED LEVELS OF DA AND NE IN PFC OF CPZ-EXPOSED MICE}

In the measurement of DA levels in PFC (Figure 4A), no significant effect was revealed by one-way ANOVA $\left[F_{(7,64)}=1.1, p>0.05\right]$. Post hoc comparisons found a significant difference between CNT and CPZ groups $(p=0.032)$. But, no differences were found between any other two groups. HAL and CLZ, but not QUE, seemed to increase DA levels in mice that consumed normal diet without $\mathrm{CPZ}$, although this effect did not reach the pre-set significant level. In the measurement of NE (Figure 4B), no significant effect was revealed by one-way ANOVA $\left[F_{(7,83)}=2.02, p>0.05\right]$. Post hoc comparisons found a significant difference between CNT and CPZ groups $(p=0.025)$. But, no differences were found between any other two groups.

\section{CLZ AND HAL BLOCK THE DECREASE IN THE AMOUNT OF MBP IN PFC OF CPZ-EXPOSED MICE}

In our recent studies (Xiao et al., 2008; Zhang et al., 2008), QUE showed protective effects on the CPZ-induced myelin sheath loss and decrease in the amount of MBP in the cerebral cortex of C57BL/6 mice. In this study, we sought to determine if HAL and CLZ have

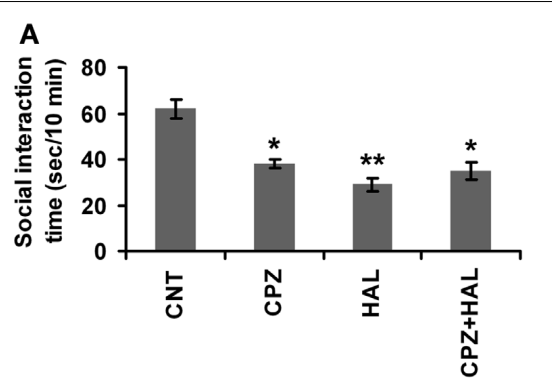

FIGURE 3 | Effects of antipsychotics on the CPZ-induced deficits in the social interaction. Control and experimentally treated $\mathrm{C} 57 \mathrm{BL} / 6$ mice were subjected to social interaction test on the same day (28th day after CPZ-exposure). (A)The data
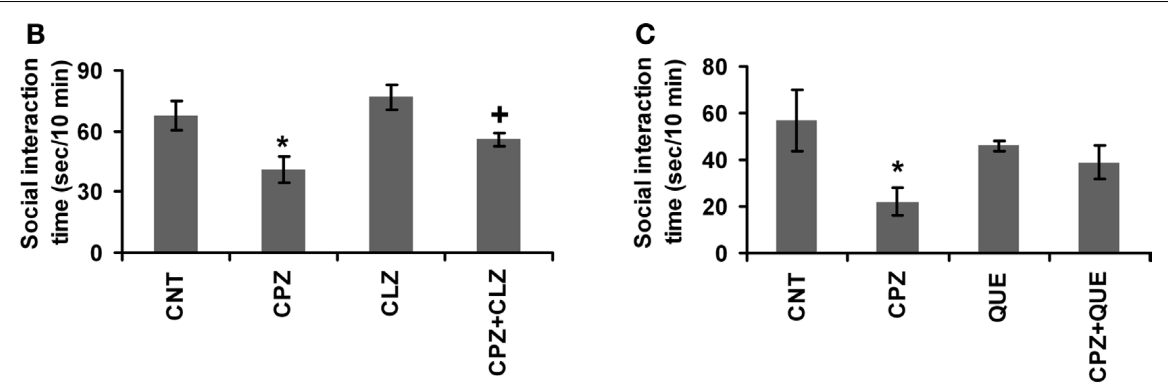

of the HAL experiment. (B) The data of the CLZ experiment. (C) The data of the QUE experiment. Data were expressed as $\mathrm{M} \pm \operatorname{SEM}\left(n=6\right.$ pairs/group). ${ }^{*} p<0.05$, ${ }^{* *} p<0.01$, compared to the CNT group; $+p<0.05$, compared to the CPZ group.

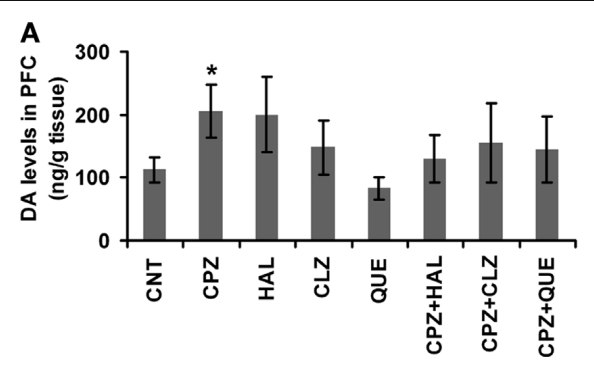

FIGURE 4 | Effects of antipsychotics on the CPZ-induced changes in levels of DA and NE in PFC. (A) Control and experimentally treated C57BL/6 mice were sacrificed on 14th day after CPZ-exposure. DA levels in PFC were measured by means of HPLC. (B) Control and experimentally treated

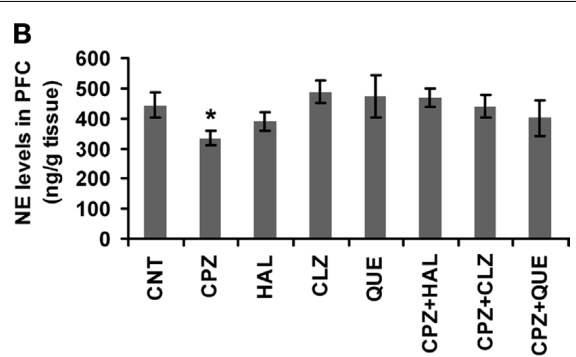

C57BL/6 mice were sacrificed on 21st day after CPZ-exposure. NE levels in PFC were measured by means of HPLC. Data were expressed as $\mathrm{M} \pm \mathrm{SEM}$ (ng/g tissue; $n=6$ to $12 /$ group). ${ }^{*} p<0.05$, compared to the CNT group. 
similar protective effects. CPZ-exposure for 42 days dramatically decreased the amount of MBP in PFC. But, this decrease was ameliorated in mice that exposed to CPZ and HAL/CLZ although those treated with the antipsychotics alone showed similar MBP immunoreactive bands to that of CNT (Figures 5A,B). The results of statisti$\mathrm{cal}$ analysis against the quantitative data were shown in the bottom panel of Figures 5C,D. For the HAL experiment (Figure 5C), twoway ANOVA revealed a significant interaction between $\mathrm{CPZ}$ and HAL treatments $\left[F_{(3,19)}=18.61, p<0.001\right]$. Post hoc comparisons revealed significant differences between $\mathrm{CNT}$ and $\mathrm{CPZ}$ groups as well as between CNT and CPZ + HAL groups. The difference between $\mathrm{CPZ}$ and $\mathrm{CPZ}+\mathrm{HAL}$ was also significant. For the CLZ experiment (Figure 5D), two-way ANOVA revealed a significant interaction between CPZ and CLZ treatments $\left[F_{(3,19)}=10.74, p<0.001\right]$. Post hoc comparisons revealed significant differences between CNT and $\mathrm{CPZ}$ groups as well as between CNT and CPZ+CLZ groups. The difference between $\mathrm{CPZ}$ and $\mathrm{CPZ}+\mathrm{CLZ}$ was also significant.

\section{CLZ AND QUE, BUT NOT HAL, PROTECT THE WHITE MATTER IN CP OF CPZ-EXPOSED MICE}

In our recent study (Yang et al., 2009), CPZ-exposed mice showed obvious myelin sheath loss in the ventral and lateral parts of $\mathrm{CP}$, where smaller fiber tracts are located. In this study, we sought to examine effects of antipsychotics on the CPZ-induced white matter damage. Similar to our recent study, myelin sheath loss was seen in CP of CPZ-exposed mice and this CPZ-induced white matter damage was ameliorated by CLZ and QUE, but not HAL (Figures 6A-C). Quantitative measurement and comparisons confirmed the morphological observations (Figures 6D-F). These results suggest that CLZ and QUE, but not HAL, protect the white matter in $\mathrm{CP}$ of mice exposed to CPZ. To further confirm these effects, Western-blot analysis was performed to measure the amount of MBP in CP. Significant decreases in the amount of
MBP in CP were seen in all experiments (Figure 7). In the HAL experiment (Figures 7A,D), this decrease in the amount of MBP was not affected by HAL as the CPZ + HAL group showed a comparable amount of MBP to that of the CPZ group. In the CLZ experiment (Figures 7B,E), this decrease in the amount of MBP was significantly ameliorated by CLZ as the CPZ + CLZ group showed a higher level of MBP compared to the CPZ group. QUE showed a similar protective effect (Figures 7C,F) as CLZ did. In addition, we did similar analyses (immunohistochemistry and Western-blot) against the hippocampus and obtained the similar results supporting the protective effects of CLZ and QUE on the CPZ-induced white matter damage (not shown).

\section{DISCUSSION}

Although many animal models of schizophrenia have been developed, no one could mimic the neuropathological feature of white matter abnormalities in schizophrenic brains. For the first time, a new animal model has been established in this study which shows white matter alterations in PFC, CP and the hippocampus, in addition to the abnormal behaviors mimicking the heterogeneity of schizophrenia symptoms. These behavioral changes include PPI deficits (Figure 1), a lower spontaneous alternation in the Y-maze (Figure 2), and less time in social interaction (Figure 3). Furthermore, HAL and SGAs showed different effects on the CPZinduced behavioral and neurobiological changes, which remind us of the different therapeutic effects of these antipsychotics in treating patients with schizophrenia.

CPZ-treated mice showed PPI deficits while they had higher levels of DA in their PFC, suggesting an association between these two variables. In support of the existence of this association, DA levels in CPZ-treated mice returned to CNT levels when PPI deficits disappeared (Xu et al., 2009). Furthermore, all antipsychotics tested in this study completely blocked the CPZ-induced

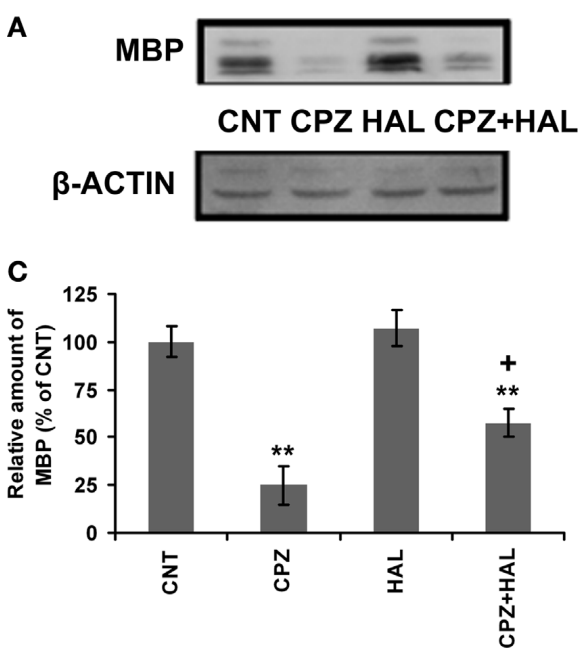

FIGURE 5 | Effects of HAL and CLZ on the CPZ-induced decrease in MBP in PFC. Control and experimentally treated C57BL/6 mice were sacrificed on 43rd day after CPZ-exposure. The PFC was dissected out of the brain and processed for Western-blot analysis to measure MBP levels. The upper photographs (A) and (B) are representative Western-blots from HAL and CLZ
B

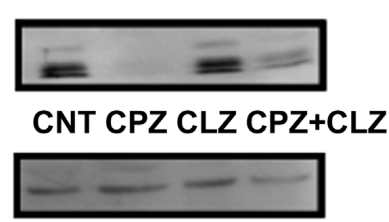

D

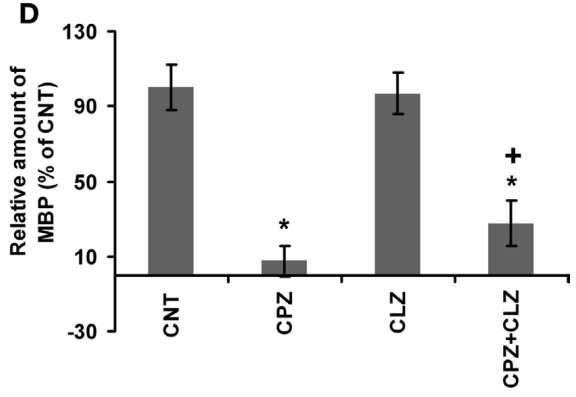

experiments, respectively. The bar charts (C) and (D) in the bottom panel are the statistical results of the amount of MBP relative to $\beta$-actin in the same corresponding lanes as labeled. Data were expressed as $\mathrm{M} \pm \mathrm{SEM}(n=6 /$ group). ${ }^{*} p<0.05,{ }^{*} p<0.01$, compared to the CNT group. $+p<0.05$, compared to the CPZ group. 


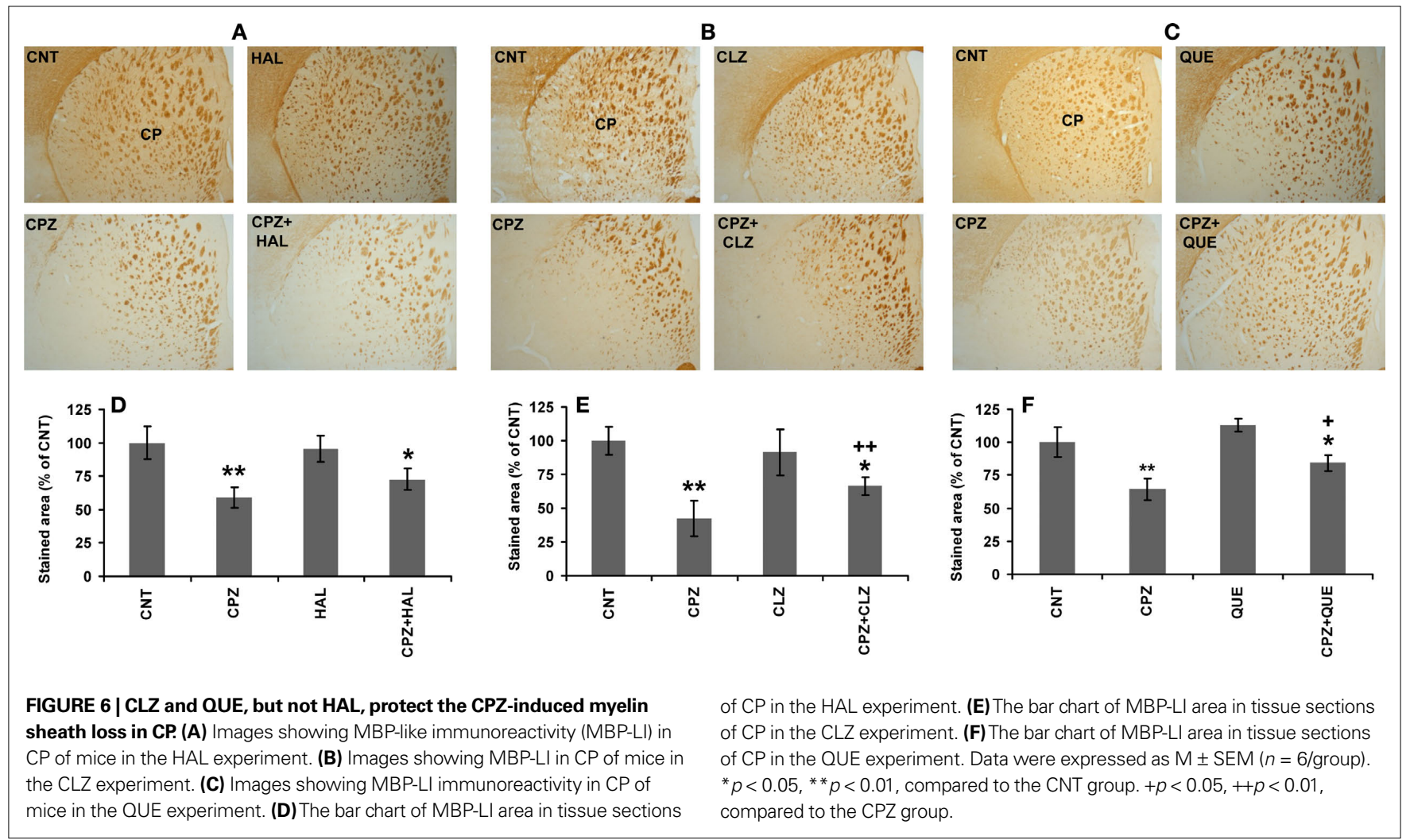

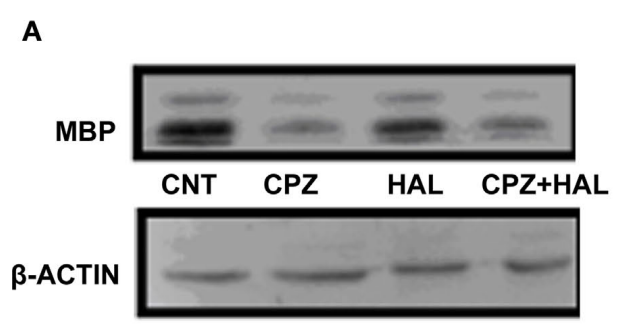

D

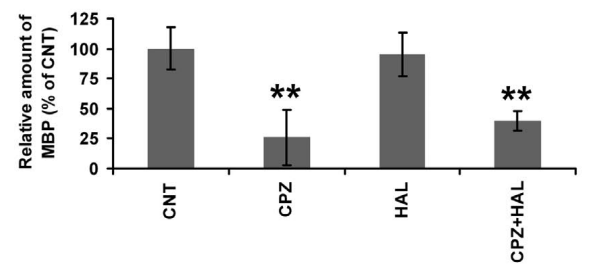

FIGURE 7 | Effects of antipsychotics on the CPZ-induced decrease in MBP in CP. Control and experimentally treated C57BL/6 mice were sacrificed on 43rd day after CPZ-exposure. The CP was dissected out of the brain and processed for Western-blot analysis to measure MBP levels. The upper photographs $(\mathbf{A}-\mathbf{C})$ are representative Western-blots from the HAL, CLZ, and

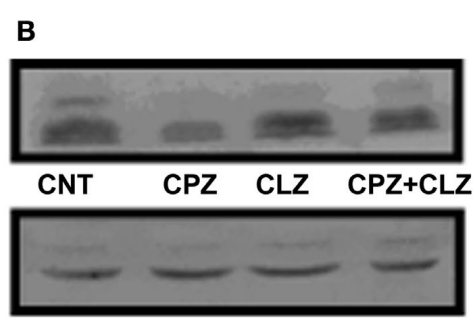

E

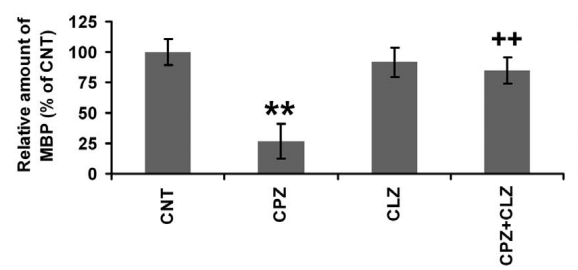

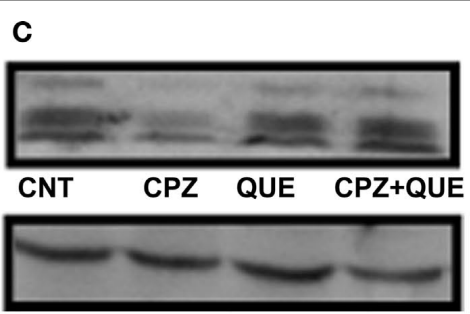

$\mathbf{F}$

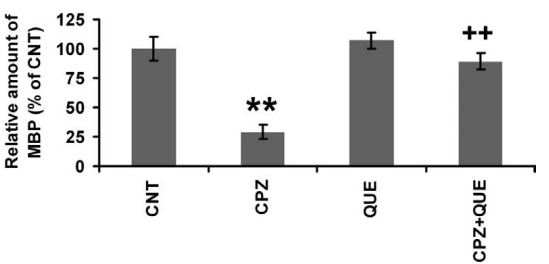

PPI deficits (Figure 1) and attenuated the CPZ-induced high levels of DA in PFC (Figure 4). These are in accordance with previous studies showing that antipsychotic agents reverse drug-induced deficits in animal models of sensorimotor gating (Olivier et al., 2001; Ouagazzal et al.,2001). Together, all these data suggest that the gating deficits in schizophrenia result from increased dopaminergic
QUE experiments, respectively. The bar charts (D-F) in the bottom panel are the statistical results of the amount of MBP relative to $\beta$-actin in the same corresponding lanes as labeled. Data were expressed as $\mathrm{M} \pm \operatorname{SEM}(n=6$ ) group). ${ }^{*} p<0.01$, compared to the CNT group; $++p<0.01$, compared to the CPZ group. neurotransmission in the brain, a proposal supported by a previous study that implicates forebrain DA over-activity in the etiology of reduced PPI (Swerdlow et al., 1986). However, it should be noted that, the antipsychotics alone did not decrease DA levels in PFC. Instead, HAL and CLZ seemed to increase DA levels. Therefore, it may be interpreted that antipsychotics improved PPI deficits 
mainly by blocking DA receptors (D2 receptors, most likely). This interpretation is in line with the evidence that the administration of DA agonists can disrupt PPI in humans (Hutchison and Swift, 1999; Swerdlow et al., 2003) and in animals (Mansbach et al., 1988; Swerdlow et al., 1990).

In a previous study, amphetamine reduced spontaneous alternation dose-dependently and HAL pretreatment inhibited this effect (Oades et al., 1985), suggesting the involvement of central DA activity in the performance of mice in Y-maze test. In our recent study (Xu et al., 2009), a lower spontaneous alternation in the Y-maze was found in CPZ-treated mice starting from 14th day after CPZ treatment when DA levels increased in PFC and remained throughout the whole 6-weeks experimental period. In the present study, HAL treatment for 21 days attenuated the CPZ-induced lower spontaneous alternation; but when the same HAL treatment continued for 42 days this attenuating effect disappeared. Moreover, HAL alone (for 42 days) caused a lower spontaneous alternation in control mice (Figure 2A). Together, all these results provide further evidence for the involvement of DA in the performance of mice in Y-maze test. In addition, the observation of the present study that HAL treatment for 21 days, but not for 42 days, attenuated the $\mathrm{CPZ}$-induced decrease in spontaneous alternation may be related to a possible D2 receptor up-regulation achieved by chronic (for 42 days) administration of HAL. The D2 receptor upregulation caused by chronic antipsychotic exposure was described in both animals (Burt et al., 1977; Ginovart et al., 2009) and patients with schizophrenia (Lee et al., 1978; Mackay et al., 1982) and now is thought to be responsible for loss of efficacy of antipsychotics. CLZ and QUE completely blocked the CPZ-induced lower spontaneous alternation in the Y-maze (Figure 2) and did not show the loss of efficacy as HAL did. This is consistent with their lower affinities for D2 receptors (Kasper et al., 1999).

The CPZ-treated mice did not show deficit in social interaction until 4th week after CPZ-exposure when the loss of myelin sheath and MBP became evident (Xu et al., 2009), suggesting normal performance in social interaction relies on intact white matter. In support of this notion, low sociability is associated with reduced size of the corpus callosum in the mouse (Fairless et al., 2008). Although it is now unclear how white matter damage impairs the social interaction in CPZ-treated mice, some clues may be drawn from the following findings of the present study: first, HAL did not improve the performance of CPZ-treated mice in social interaction test (Figure 3A) whereas it mitigated the CPZ-induced MBP decrease in PFC (Figure 5); second, the myelin sheath loss in CPZ + CLZ and CPZ + QUE groups were still obvious (Figure 6) but their performance in social interaction test was comparable to that of the CNT group (Figure 3). These results suggest a reasonable interpretation that the improvement of the performance of CPZtreated mice in the social interaction by CLZ and QUE is mainly due to the actions of these two drugs on various neurotransmitter systems that had become dysfunctional following white matter damage. HAL acts on D2 receptors mainly while CLZ and QUE are broad-acting agents with affinities for receptors of a variety of neurotransmitters; therefore, HAL did not have the same beneficial effects on the social interaction deficit as CLZ and QUE did. In line with this interpretation, a recent study (Roy et al., 2007) reported increased levels of DA receptors in the transgenic mice with OL alterations. In future studies, we will address which and how neurotransmitter systems become dysfunctional following white matter damage.

Consistent with previous studies (Prohaska and Bailey, 1993; Xu et al., 2009), CPZ-treated mice had lower NE levels in PFC while they showed abnormal behaviors, suggesting the involvement of $\mathrm{NE}$ system in the CPZ-induced abnormal behaviors. The decrease in NE levels may be related to the inhibition of the activity of DA $\beta$ hydroxylase $(\mathrm{D} \beta \mathrm{H})$, a cupric enzyme which catalyzes the synthesis of NE from DA. In fact, C57BL/6 mice fed the CPZ-containing diet for 21 days showed lower activity of $\mathrm{D} \beta \mathrm{H}$ in the hippocampus and $\mathrm{PFC}$ as compared to normal controls (Xu et al., 2009). More interestingly, the CPZ-induced decrease in NE levels was blocked by the antipsychotics tested in this study (Figure 4). These results are in line with the following previous findings: antipsychotics including HAL and SGAs dose dependently increase NE release in the rat medial PFC and hippocampus (Westerink et al., 1998); systemic administration of HAL and CLZ increases the firing rate of NE neurons in locus coeruleus (Nilsson et al., 2005), the primary source of the noradrenergic innervations of the forebrain; and QUE increases levels of NE in both PFC and the caudate nucleus (Pira et al., 2004).

Both CLZ and HAL ameliorated the decrease in the amount of MBP in PFC of mice exposed to CPZ (Figure 5) and the same drugs also blocked the CPZ-induced high levels of DA in the same brain region (Figure 4). This association between normalized DA levels and ameliorated MBP changes in PFC implies that high levels of DA may contribute to the CPZ-induced white matter damage in PFC. In support of this speculation, previous studies have shown that high levels of DA act as an endogenous neurotoxin (Bozzi and Borrelli, 2006) and may damage OLs by increasing free radical generation (Khorchid et al., 2002). The mitochondrial impairment and metabolic stress, in turn, increase DA efflux (Moy et al., 2007). Therefore, it is reasonable to suggest a mechanism that CLZ and HAL act on DA system (DA levels and/or DA receptors) in PFC thus protect the white matter there of CPZ-exposed mice. By this mechanism, these two drugs block the PPI deficit and lower spontaneous alternation in CPZ-treated mice.

Both CLZ and QUE, but not HAL, protect the white matter in CP of mice exposed to CPZ (Figures 6 and 7), suggesting that additional mechanisms are involved in the protective effect of these two SGAs besides the anti-dopaminergic action shared by all the three antipsychotics as discussed above. Although this study did not address what are the exact mechanisms, our recent work on QUE allows us to suggest a most likely mechanism that QUE protects $\mathrm{CPZ}$-induced white matter damage mainly by its antioxidative actions. Our recent findings supporting the antioxidative actions of QUE include: (1) QUE scavenges hydroxyl radical produced in the Fenton reaction and in the Aß25-35 solution (Xu et al., 2008); (2) QUE prevents PC12 cell death caused by increased intracellular reactive oxygen species and calcium (Wang et al., 2005); and (3) QUE attenuates the CPZ-induced decrease in the activity of SOD1 in the cerebral cortex of mice (Zhang et al., 2008). This suggested mechanism is also in accordance with the mechanism by which CPZ damages OLs. That is, CPZ decreases activities of cupric enzymes, such as SOD1 (Ljutakova and Russanov, 1985) and cytochrome oxidase (Wakabayashi et al., 1978), thus causes oxidative stress and leads to OLs death and 
demyelination in the brain (Mason et al., 2004; Copray et al., 2005). CLZ may possess a similar mechanism because this antipsychotic acts as a very good antioxidant in lipid peroxidation produced in microsomal membranes (Dalla Libera et al., 1998).

In summary, CPZ acts on cupric enzymes, including SOD1, cytochrome oxidase, and $\mathrm{D} \beta \mathrm{H}$ thus causes oxidative stress and increases DA levels in certain brain regions such as PFC (Figure 4). High levels of DA results in abnormal behaviors such as PPI deficits (Figure 1), and exacerbates the oxidative stress, which is fatal to OLs. The loss of myelin sheath integrity (Figure 6) not only affects the communication between different brain regions and may impair the coordination and balance of different neurotransmitter systems in the brain. As a result of these changes, CPZ-treated mice show more behavioral alterations including the social interaction deficit and impairment in spatial working memory (Figures $\mathbf{2}$ and $\mathbf{3}$ ).

\section{REFERENCES}

Abi-Dargham, A., Rodenhiser, J., Printz, D., Zea-Ponce, Y., Gil, R., Kegeles, L. S., Weiss, R., Cooper, T. B., Mann, J. J., Van Heertum, R. L., Gorman, J. M., and Laruelle, M. (2000). Increased baseline occupancy of $\mathrm{D} 2$ receptors by dopamine in schizophrenia. Proc. Natl. Acad. Sci. U.S.A. 97, 8104-8109.

Ardekani, B. A., Nierenberg, J., Hoptman, M. J., Javitt, D. C., and Lim, K. O. (2003). MRI study of white matter diffusion anisotropy in schizophrenia. Neuroreport 14, 2025-2029.

Bai, O., Chlan-Fourney, J., Bowen, R., Keegan, D., and Li, X. M. (2003). Expression of brain-derived neurotrophic factor mRNA in rat hippocampus after treatment with antipsychotic drugs. J. Neurosci. Res. 71, 127-131.

Bai, O., Zhang, H., and Li, X. M. (2004). Antipsychotic drugs clozapine and olanzapine upregulate bcl-2 mRNA and protein in rat frontal cortex and hippocampus. Brain Res. 1010, 81-86.

Bassett, A. S., Chow, E. W., AbdelMalik, P., Gheorghiu, M., Husted, J., and Weksberg, R. (2003). The schizophrenia phenotype in 22q11 deletion syndrome. Am. J. Psychiatry 160, 1580-1586.

Boucher, A. A., Arnold, J. C., Duffy, L., Schofield, P. R., Micheau, J., and Karl, T. (2007). Heterozygous neuregulin 1 mice are more sensitive to the behavioural effects of Delta9-tetrahydrocannabinol. Psychopharmacology (Berl.) 192, 325-336.

Bozzi,Y., and Borrelli,E. (2006). Dopamine in neurotoxicity and neuroprotection: what do D2 receptors have to do with it? Trends Neurosci. 29, 167-177.

Burt, D. R., Creese, I., and Snyder, S. H. (1977). Antischizophrenic drugs: chronic treatment elevates dopamine receptors binding in brain. Science 196, 326-328.
Carpenter, W. T., and Buchanan, R. W. (1994). Schizophrenia. N. Engl. J. Med. 330, 681-690.

Copray,J.C., Küst, B. M., Mantingh-Otter, I., and Boddeke, H.W. (2005).p75NTR independent oligodendrocyte death in cuprizone-induced demyelination in C57BL/6 mice. Neuropathol. Appl. Neurobiol. 31, 600-609.

Dalla Libera, A., Scutari, G., Boscolo, R., Rigobello, M. P., and Bindoli, A. (1998). Antioxidant properties of clozapine and related neuroleptcs. Free Radic. Res. 29, 151-157.

Davis, K. L., Stewart, D. G., Friedman, J. I., Buchsbaum, M., Harvey, P. D., Hof, P. R., Buxbaum, J., and Haroutunian, V. (2003). White matter changes in schizophrenia. Arch. Gen. Psychiatry $60,443-456$.

Egashira, N., Tanoue, A., Matsuda, T., Koushi, E., Harada, S., Takano, Y., Tsujimoto, G., Mishima, K., Iwasaki, K., and Fujiwara, M. (2007). Impaired social interaction and reduced anxietyrelated behavior in vasopressin $\mathrm{Vla}$ receptor knockout mice. Behav. Brain Res. 178, 123-127.

Ellenbroek, B. A. (1993). Treatment of schizophrenia: a clinical and preclinical evaluation of neuroleptic drugs. Pharmacol. Ther. 57, 1078.

Fairless, A. H., Dow, H. C., Toledo, M. M., Malkus, K. A., Edelmann, M., Li, H., Talbot, K., Arnold, S. E., Abel, T., and Brodkin, E. S. (2008). Low sociability is associated with reduced size of the corpus callosum in the BALB/cJ inbred mouse strain. Brain Res. 1230, 211-217.

Franke, P., Maier, W., Hain, C., and Klingler, T. (1992). Wisconsin Card Sorting Test: an indicator of vulnerability to schizophrenia? Schizophr. Res. 6, 243-249.

Ginovart, N., Wilson, A. A., Hussey, D., Houle, S., and Kapur, S. (2009). D2-receptor upregulation is dependent upon temporal course

HAL has high affinities for D2 receptors, thus improves the CPZinduced PPI deficit and impairment in spatial working memory. In addition to DA system, CLZ and QUE act on more neurotransmitter systems thus improve more behavioral changes seen in CPZtreated mice. Moreover, these two SGAs have antioxidant properties thus protect mice against the $\mathrm{CPZ}$-induced white matter damage (Figures 5,6 and 7). This protective action may also contribute to the drugs' effects on the social interaction deficit and impairment in spatial working memory in CPZ-treated mice.

\section{ACKNOWLEDGMENTS}

This study was supported by a start-up fund from the School of Medicine, Southern Illinois University Carbondale and a faculty seed fund from the University to Haiyun Xu. Drs Gregory Rose and Rich Clough read the manuscript.

of D2-occupancy: A longitudinal $[11 \mathrm{C}]$-raclopride PET study in cats. Neuropsychopharmacology 34 , 662-671.

Gregg, J. R., Herring, N. R., Naydenov, A. V., Hanlin, R. P., and Konradi, C. (2009). Downregulation of oligodendrocyte transcripts is associated with impaired prefrontal cortex function in rats. Schizophr. Res. 113 277-287.

Hakak, Y., Walker, J. R., Li, C., Wong, W. H., Davis, K. L., Buxbaum, J. D., Haroutunian, V., and Fienberg, A. A. (2001). Genome-wide expression analysis reveals dysregulation of myelination-related genes in chronic schizophrenia. Proc. Natl. Acad. Sci. U.S.A. 98, 4746-4751.

Harvey, P. D., and Keefe, R. S. E. (2001). Studies of cognitive changes in patients with schizophrenia following novel antipsychotic treatment. Am. J. Psychiatry 158, 176-184.

Hutchison, K. E., and Swift, R. (1999). Effect of D-amphetamine on prepulse inhibition of the startle reflex in humans. Psychopharmacology (Berl.) $143,394-400$.

Hyde, T.M.,Ziegler, J.C., and Weinberger, D. R. (1992). Psychiatric disturbances in metachromatic leukodystrophy: insights into the neurobiology of psychosis. Arch. Neurol. 49, 401-406.

Kane, J., Honigfeld, G., Singer, J., and Meltzer, H. (1988). Clozapine for the treatment-resistant schizophrenic: a double-blind comparison with chlorpromazine. Arch. Gen. Psychiatry 45 789-796.

Kasper, S., Hale, A., Azorin, J. M., and Möller, H. J. (1999). Benefit-risk evaluation of olanzapine, risperidone and sertindole in the treatment of schizophrenia. Eur. Arch. Psychiatry Clin. Neurosci. 249(Suppl. 2), II1-II14.

Khorchid, A., Fragoso, G., Shore, G., and Almazan, G. (2002). Catecholamine- induced oligodendrocyte cell death in culture is developmentally regulated and involves free radical generation and differential activation of caspase3. Glia 40, 283-299.

Kohler, J., Heilmeyer, H., and Volk, B. (1988). Multiple sclerosis presenting as chronic atypical psychosis. J. Neurol. Neurosurg. Psychiatr. 51, 281-284.

Kubicki,M.,McCarley, R.W., and Shenton, M. E. (2005). Evidence for white matter abnormalities in schizophrenia. Curr Opin Psychiatry 18, 121-134.

Lazar, N. L., Ragakumar, N., and Cain, D. P. (2008). Injections of NGF into neonatal frontal cortex decrease social interaction as adults: a rat model of schizophrenia. Schizophr. Bull. 34, 127-136.

Lee, T., Seeman, P., Tourtellotte, W. W., Farley, I. J., and Hornykeiwicz, O. (1978). Binding of $3 \mathrm{H}$-neuroleptics and $3 \mathrm{H}$-apomorphine in schizophrenic brains. Nature 274, 897-900.

Ljutakova, S. G., and Russanov, E. M (1985). Differences in the in vivo effects of cuprizone on superoxide dismutase activity in rat liver cytosol and mitochondrial intermembrane space. Acta Physiol. Pharmacol. Bulg. 11, 56-61.

Mackay, A. V., Iversen, L. L., Rossor, M., Spokes, E., Bird, E., Arregui,A., Creese, I., and Synder, S. H. (1982). Increased brain dopamine and dopamine receptors in schizophrenia. Arch. Gen. Psychiatry 39, 991-997.

Mansbach, R. S., Geyer, M. A., and Braff, D. I. (1988). Dopaminergic stimulation disrupts sensorimotor gating in the rat. Psychopharmacology (Berl.) 94, 507-514.

Mason, J. L., Toews, A., Hostettler, J. D., Morell, P., Suzuki, K., Goldman, J. E., and Matsushima, G. K. (2004). Oligodendrocytes and progenitors become progressively depleted within chronically demyelinated lesions. Am. J. Pathol. 164, 1673-1682. 
Matsushima, G. K., and Morell, P. (2001). The neurotoxicant, cuprizone, as a model to study demyelination and remyelination in the central nervous system. Brain Pathol. 11, 107-116.

McCullumsmith,R.E., Gupta, D., Beneyto, M., Kreger, E., Haroutunian, V., Davis, K. L., and Meador-Woodruff, J. H. (2007). Expression of transcripts for myelination related genes in the anterior cingulate cortex in schizophrenia. Schizophr. Res. 90, 15-27.

Meltzer, H. Y. (1995). Role of serotonin in the action of atypical antipsychotic drugs. Clin. Neurosci. 3, 64-75.

Moy, L. Y., Wang, S. P., and Sonsalla, P. K. (2007). Mitochondrial stress-induced dopamine efflux and neuronal damage by malonate involves the dopamine transporter. J. Pharmacol. Exp. Ther. 320, 747-756.

Nilsson, L. K., Schwieler, L., Engberg, G., Linderholm, K. R., and Erhardt, S. (2005). Activation of noradrenergic locus coeruleus neurons by clozapine and haloperidol: involvement of glutamatergic mechanisms. Int. J. Neuropsychopharmacol. 8, 329-339.

Oades, R., Taghzouti, K., Simon, H., and Le Moal, M. (1985). Dopamine-sensitive alternation and collateral behavior in a Y-maze: effects of d-amphetamine and haloperidol. Psychopharmacology (Berl.) 85, 123-128.

Olivier, B., Leahy, C., Mullen, T., Paylor, R., Groppi, V. E., Sarnyai, Z., and Brunner, D. (2001). The DBA/2J strain and prepulse inhibition of startle: a model system to test antipsychotics. Psychopharmacology (Berl.) 156, 284-290.

Ouagazzal, A. M., Jenck, F., and Moreau, J. L. (2001). Drug-induced potentiation of prepulse inhibition of acoustic startle reflex in mice: a model for detecting antipsychotic activity? Psychopharmacology 156, 273-283.

Pira, L., Mongeau, R., and Pani, L. (2004). The atypical antipsychotic quetiapine increases both noradrenaline and dopamine release in the rat prefrontal cortex. Eur. J. Pharmacol. 504, 61-64.

Prohaska, J. R., and Bailey, W. R. (1993). Persistent regional changes in brain copper, cuproenzymes and catecholamines following perinatal copper deficiency in mice. J. Nutr. 123, 1226-1234.

Reynolds, G. P. (2004). Receptor mechanisms in the treatment of schizophrenia. J. Psychopharmacol. (Oxford) 18, 340-345.

Roy, K., Murtie, J. C., El-Khodor, B. F., Edgar, N., Sardi, S. P., Hooks, B. M., Benoit-Marand, M., Chen, C., Moore, H., O’Donnell, P., Brunner, D., and Corfas, G. (2007). Loss of erbB signaling in oligodendrocytes alters myelin and dopaminergic function, a potential mechanism for neuropsychiatric disorders. Proc. Natl. Acad. Sci. U.S.A. 104, 8131-8136.

Seeman, P. (2002). Atypical antipsychotics: mechanism of action. Can. J. Psychiatry 47, 27-38.

Seeman, P.(2006). Targeting the dopamine D2 receptor in schizophrenia. Expert Opin. Ther. Targets 10, 515-531.

Shi, L., Fatemi, S. H., Sidwell, R. W., and Patterson, P. H. (2003). Maternal influenza infection causes marked behavioral and pharmacological changes in the offspring. J. Neurosci. 23, 297-302.

Swerdlow, N. R., Braff, D. L., Geyer, M. A., and Koob, G. F. (1986). Central dopamine hyperactivity in rats mimics abnormal acoustic startle response in schizophrenics. Biol. Psychiatry 21, 23-33.

Swerdlow, N. R., Braff, D. L., Masten, V. L., and Geyer, M. A. (1990). Schizophrenic-like sensorimotor gating abnormalities in rats following dopamine infusion into the nucleus accumbens. Psychopharmacology (Berl.) 101, 414-420.

Swerdlow, N.R., Stephany, N., Wasserman, L. C., Talledo, J., Shoemaker, J., and Auerbach, P. P. (2003). Amphetamine effects on prepulse inhibition across- species: replication and parametric extension. Neuropsychopharmacology 28, 640-650.

Wakabayashi, T., Asano, M., Ishikawa, K., and Kishimoto, H. (1978). Mechanism of the formation of megamitochondria by copper-chelating agents. V. Further studies on isolated megamitochondria. Acta Pathol. Jpn. 28 215-223.

Wang, H., Xu, H., Dyck, L. E., and Li, X. M. (2005). Olanzapine and quetiapine protect PC12 cells from beta-amyloid peptide (25-35)-induced oxidative stress and the ensuing apoptosis. $J$. Neurosci. Res. 81, 572-580.

Weinberger, D. R. (1987). Implications of normal brain development for the pathogenesis of schizophrenia. Arch. Gen. Psychiatry 44, 660-669.

Westerink, B. H., de Boer, P., de Vries, J. B. Kruse, C. G., and Long, S. K. (1998) Antipsychotic drugs induce similar effects on the release of dopamine and noradrenaline in the medial prefrontal cortex of the rat brain. Eur. J. Pharmacol. 361, 27-33.

Woodward, N. D., Purdon, S. E., Meltzer H. Y., and Zaid, D. H. (2005). A metaanalysis of neuropsychological change to clozapine, olanzapine, quetiapine, and risperidone in schizophrenia. Int J. Neuropsychopharmacol. 8, 1-16.

Xiao, L., Xu, H., Zhang, Y., Wei, Z., He, J. Jiang, W., Li, X., Dyck, L. E., Devon, R. M., Deng, Y., and Li, X. M. (2008). Quetiapine facilitates oligodendrocyte development and prevents mice from myelin breakdown and behavioral changes. Mol. Psychiatry 13, 697-708.

Xu, H., Qing, H., Lu, W., Keegan, D. Richardson, J.S., Chlan-Fourney, J., and Li, X.M. (2002). Quetiapine attenuates the immobilization stress-induced decrease of brain-derived neurotrophic factor expression in rat hippocampus. Neurosci. Lett. 321, 65-68.

Xu, H., Wang, H., Zhuang, L., Yan, B., Yu, Y., Wei, Z., Zhang, Y., Dyck, L. E. Richardson, S. J., He, J., Li, X., Kong, J., and Li, X. M. (2008). Demonstration of an anti-oxidative stress mechanism of quetiapine: implications for the treatment of Alzheimer's disease. FEBS J. 275, 3718-3728.

Xu, H., Yang, H. J., Zhang, Y., Clough, R., Browning, R., and Li, X. M. (2009). Behavioral and neurobiological changes in C57BL/6 mice exposed to cuprizone. Behav. Neurosci. 123, 418-429.

Yang, H. J., Wang, H., Zhang, Y., Xiao, L., Clough, R.W., Browning, R., Li, X. M., and $\mathrm{Xu}, \mathrm{H}$. (2009). Region-specific susceptibilities to cuprizone-induced lesions in the mouse forebrain: Implications for the pathophysiology of schizophrenia. Brain Res. 1270, 121-130.

Zhang, Y., Xu, H., Jiang, W., Xiao, L., Yan, B., He, J., Wang, Y., Bi, X., Li, X., Kong, J., and Li, X. M. (2008). Quetiapine alleviates the cuprizone-induced white matter pathology in the brain of C57BL/6 mouse. Schizophr. Res. $106,182-191$.

Conflict of Interest Statement: All authors declare that the research was conducted in the absence of any commercial or financial relationships that could be construed as a potential conflict of interest.

Received: 09 January 2010; paper pending published: 23 January 2010; accepted: 08 February 2010; published online: 18 March 2010

Citation: Xu H, Yang H-J, McConomy B, Browning R and LiX-M (2010) Behavioral and neurobiological changes in C57BL/6 mouse exposed to cuprizone: effects of antipsychotics. Front. Behav. Neurosci. 4:8. doi: 10.3389/fnbeh.2010.00008

Copyright (C) $2010 \mathrm{Xu}$, Yang, McConomy, Browning and $\mathrm{L} i$. This is an open-access article subject to an exclusive license agreement between the authors and the Frontiers Research Foundation, which permits unrestricted use, distribution, and reproduction in any medium, provided the original authors and source are credited. 